\title{
Structure of Turbulent Vortices in a Compound Channel
}

\author{
Alfonsas Rimkus \\ Water Research Institute of Lithuanian Aleksandras Stulginskis University, LT-58102, Vilainiai, Kedainiai \\ Lithuania, e-mail: alfonsas.rimkus@hidro.lzuu.lt
}

(Received April 26, 2012; revised January 14, 2013)

\begin{abstract}
In spite of many investigations performed on turbulent flows, their structure has not yet been sufficiently explored. The difficulty is that, when a detailed picture of the velocity field is necessary, the widely employed Particle Image Velocity (PIV) method can provide photos covering only a short interval of flow, which cannot include the largest structures of turbulent flow, and consequently these structures cannot be investigated. In this study, the author tried to obtain necessary data about the processes occurring in the flow by analyzing instantaneous velocity measurements carried out by 3D means. A measurement at the points of a flow cross-section takes at least 1 minute. During this time all vortex structures, including the largest, occur repeatedly many times and can be studied. The analysis of such measurements was the aim of this article. The process of the generation of vortices at the bottom and their further development, including the conditions of the development of the largest vortices, has been investigated. The results of these investigations are discussed in this article.
\end{abstract}

Key words: hydraulic investigations, turbulent vortices, measurement analysis, turbulent structure.

\section{Introduction}

Despite abundant literature devoted to turbulent flow investigations, the process of the generation and further development of turbulent vortices has not yet been sufficiently expolored, although the structure of these vortices had already been investigated for many years now (Vanoni and Nomicos 1959, Klaven 1966, Grishanin, 1969, Cuthbertson and Ervin 1999, Nezu and Azuma 2004). The structure of turbulent vortices revealed by Vanoni's experiments, i.e. the distribution of vortices in straight experimental channels, is presented in Fig. 1. There are two main types of such vortices: (1) bottom vortices that form as a result of high local velocity gradients existing near the bottom, and (2) large whirlpools developing along the whole depth of flow.

Bottom vortices often originate as a result of the separation of flow from the bottom unevenness; therefore they are displaced along the flow accidentally. They do not exist for a long time, and only a few of them grow to 0.3 of water depth (Cuthbertson and Ervin 1999). In contrast, the already developed large whirlpools are regular, they 


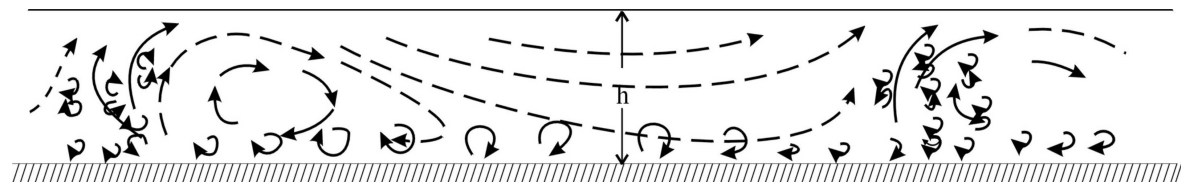

Fig. 1. Distribution of turbulent vortices according to Vanoni and Nomicos (1959)

recur in the flow periodically and seem to be stable. The distance between them, as it can be seen in Fig. 1, is equal to about 6 water depths. This is a characteristic kind of vortices, described by Yalin (1992) as "the largest turbulent vortices". In this article they are called "macro whirlpools" for short.

The Particle Image Velocimetry (PIV) photography of turbulent flow obtained in Klaven's (1966) experiments is presented in Fig. 2. Two macro whirlpools and several bottom vortices are visible in this photo. The height of the first is equal to about 0.7 of water depth. The second is more developed, as these macro whirlpools flowing along the flow are still growing. Therefore the second whirlpool includes more bottom vortices. Eddies existing near the bottom are not visible in this photo, as the velocity of the photo-camera $(21.5 \mathrm{~cm} / \mathrm{sec})$ was too large to record it.

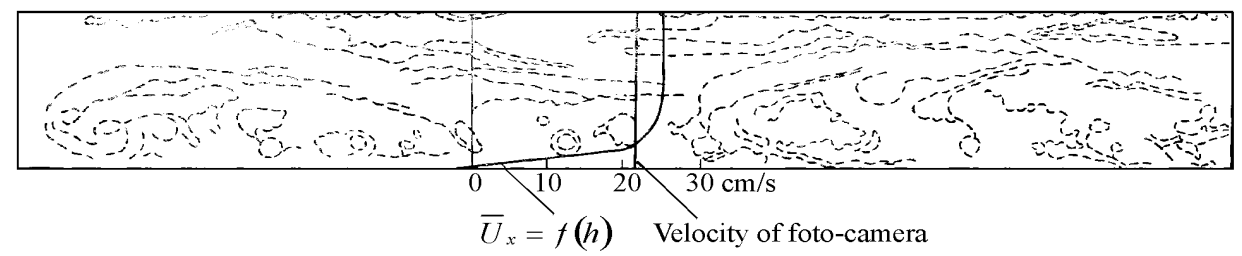

Fig. 2. Vector field of flow velocities according to Klaven's investigations (1966)

These regular macro whirlpools have also been revealed by field investigations in a straight interval of the Nevëžis river in Lithuania (Rimkus and Vaikasas 2001). The distance between them was equal to 6 water depths, as well.

The main part of turbulence is created by bottom vortices. Macro whirlpools lift water volume containing bottom vortices up to the water surface of flow. Thus they help create turbulence throughout the flow volume. In the case of suspension flow, they also lift sediments raised by bottom vortices to the water surface. Consequently, they are an important kind of turbulent vortices, which has not yet been sufficiently investigated.

Vanoni's and Klaven's velocity measurements were performed by the 2-D PIV method. Measurements with 3-D means also showed the existence of transversal components of velocity fluctuations (Breuer and Haenel 1989, Robinson 1991, Adrian et al 2000, Nezu and Nakahawa 1993, Widera et al 2009, and others). These fluctuations are formed because the intervals of the axis of bottom vortices are not long, and their 
ends are bent towardsthe bottom. Bottom vortices thus acquire rotating legs in which the transversal velocity components are formed.

During the further development of bottom vortices, these legs are usually moved to the bottom by flow velocities rapidly growing along the water depth. Consequently, these vortices become hairpin-like structures (Adrian et al 2000). The legs, moved to the bottom, become quite long form the intervals of longitudinal spiral vortices. Bottom vortices are usually formed in groups. Such vortices are discussed in many studies (Adrian et al 2000, Albayrak and Lemmin 2007, 2008, Rodriguez and Garcia 2008, and others).

The difficulty of employing the PIV method for the investigation of flow structures is that, whenever a detailed picture of flow is necessary for investigations, the photos obtained by this method record only a short interval of the flow, which cannot include even a single macro whirlpool, and thus such whirlpools cannot be investigated. Macro whirlpools were observed by the PIV method in only two of the above-mentioned studies, performed in a long interval of straight rectangular channels. The development of both these kinds of turbulent vortices has not been sufficiently investigated, because the measurement by the PIV method takes only a short time. In this study, the author tried to obtain some new data about processes occurring in the flow by analyzing instantaneous velocity measurements carried out by $3 \mathrm{D}$ means. The measurement of velocities in vertical cross-sections of the flow takes at least 1 minute, in which macro whirlpools, in their entire length, move through the measurement section. Periodically they recur along with other vortices. Thus it is possible to treat measurement chronograms as a picture with fixed vortices in a sufficiently long interval of a developed flow. Velocity measurements in the whole cross-section can provide a picture of the structure of all turbulent vortices in the flow. The aim of this study was therefore to investigate vortex properties by analyzing such measurement data. The paper discusses the results of such analysis for a compound channel.

\section{Laboratory Investigations}

The measurements analyzed here were performed in the Hydraulic Laboratory of the Department of Hydraulic Structures, Faculty of Environmental Science, of Warsaw Agricultural University in a concrete channel $16 \mathrm{~m}$ long $2.1 \mathrm{~m}$ wide with a three-component acoustic Doppler velocity meter at a frequency of $25 \mathrm{~Hz}$. Each measurement lasted $6.0 \mathrm{~min}$ (9000 measurement steps). The cross-section of the channel and the displacement of measurement verticals are shown in Fig. 3.

The bed slope of the channel was $0.05 \%$. The water depth was $H=28.3 \mathrm{~cm}$, and water discharge $Q=95.2 \mathrm{l} / \mathrm{s}$. The surface of the main channel bed was smooth; the floodplains and sloping banks were covered in cement with grains of 0.5 to $1 \mathrm{~cm}$ in diameter.

These measurement data have already been analyzed for investigation of turbulence (Czernuszenko et al 2007, Chernuszenko 2002, Czernuszenko and Rowiński 


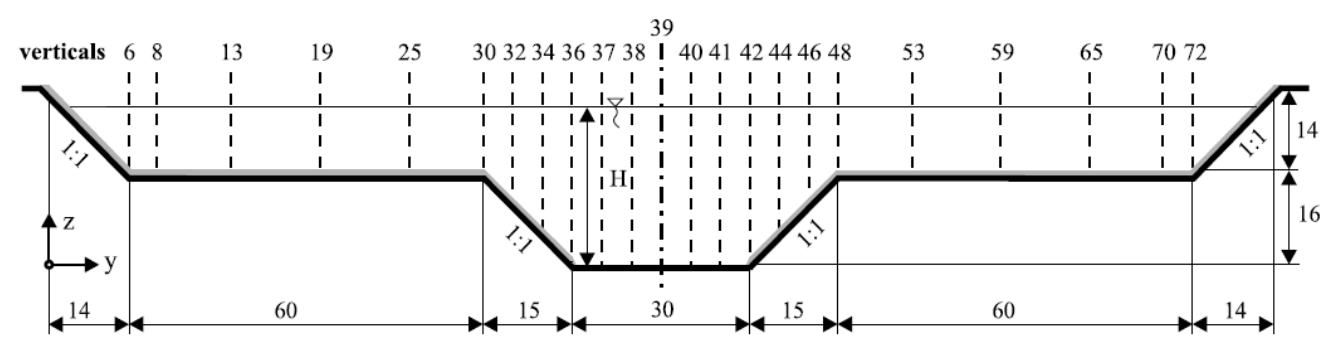

Fig. 3. Cross-section of the experimental channel and the location of measurement verticals. The measurements of the model are given in $\mathrm{cm}$

2008). The distribution of turbulent intensities, Reynolds stresses, autocorrelation functions, turbulent scales, and energy spectra were investigated. In this article these measurement data are employed for the study of vortex structures: their generation, development and interaction in the process of flow structure formation. Such data make it possible to better understand these processes and peculiarities of the flow, as the above flow characteristics depend on formed vortex structures.

\section{The Analysis of Velocity and Turbulence Intensity Distribution in the Main Channel}

The velocity distribution and its fluctuations were analyzed in measurement verticals 39, 38, 37 and 36 of the main channel, which are shown in figures 4a, 4b, 5a and 5b. These fluctuations represent the turning intensity of bottom vortices and their further development.

Fig. 4 shows the average values of longitudinal $\bar{u} / u_{*}$, vertical $\bar{v} / u$ and transversal $\bar{w} / u$ velocities, calculated from these data, where $u, v, w$ are instantaneous velocities measured in the channel. Fig. 5 illustrates turbulence intensity values: $\sqrt{\left(\overline{u-\bar{u})^{2}}\right.} / u_{*}$ - longitudinal, $\sqrt{\overline{(v-\bar{v})^{2}}} / u_{*}-$ vertical and $\sqrt{\overline{(w-\bar{w})^{2}}} / u_{*}-$ transversal. The shear velocity: $u_{*}=\sqrt{g R i}=2.29 \mathrm{~cm} / \mathrm{s}$.

\subsection{Generation and Development of Bottom Vortices}

The points at which bottom vortices are generated are visible in the turbulent intensity profiles shown in Fig. 5a and 5b. Particularly large fluctuations of both longitudinal and vertical velocities appear in verticals 38 and 39 at a height of $3 \mathrm{~cm}$ from the bottom ( 0.1 of water depth $h)$, exactly where bottom vortices are generated. In this case the measurement point happened to coincide with that place. It should be noted that such high fluctuations near the bottom were not observed, for example, in Cellino's investigations (1999), performed in a straight flume with high bottom roughness, where bottom eddies were mostly generated at the protuberances of the bottom. Then at a height of $0.1 \mathrm{~h}$ the velocities were only slightly increased. In our model, the laminar 

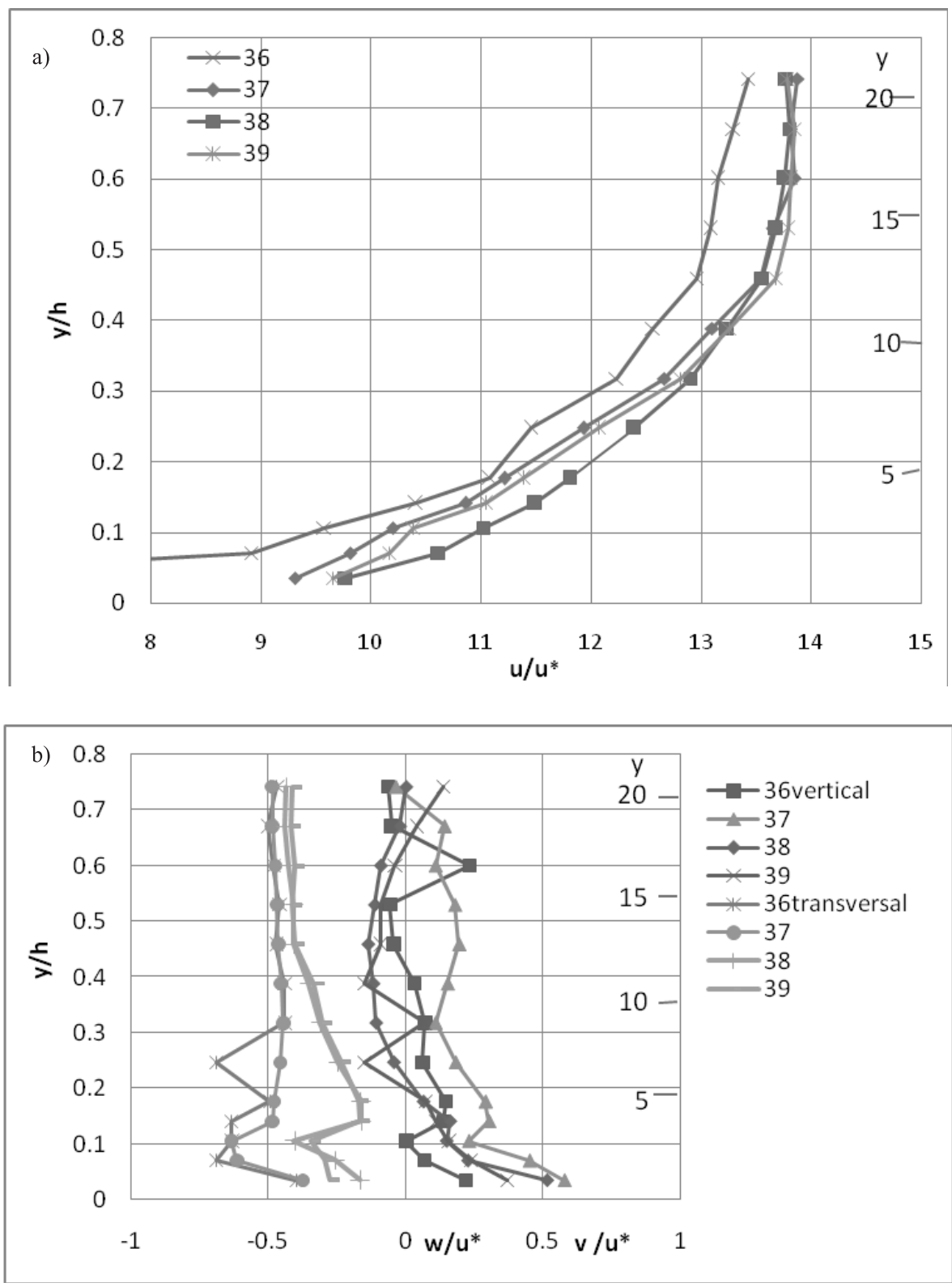

Fig. 4. Velocity profiles in measurement verticals 39, 38, 37, 36; a - of longitudinal velocities, $\mathrm{b}$ - of transversal and vertical velocities

layer was situated close to the flat bottom, so conditions for vortex generation and critical velocity module gradients were different. In this model, velocity fluctuations at the points of their generation are not very large in the valley, where the flow bottom is rough. It is possible that more information about the process of vortex generation could be obtained if more measurement points were established there. 

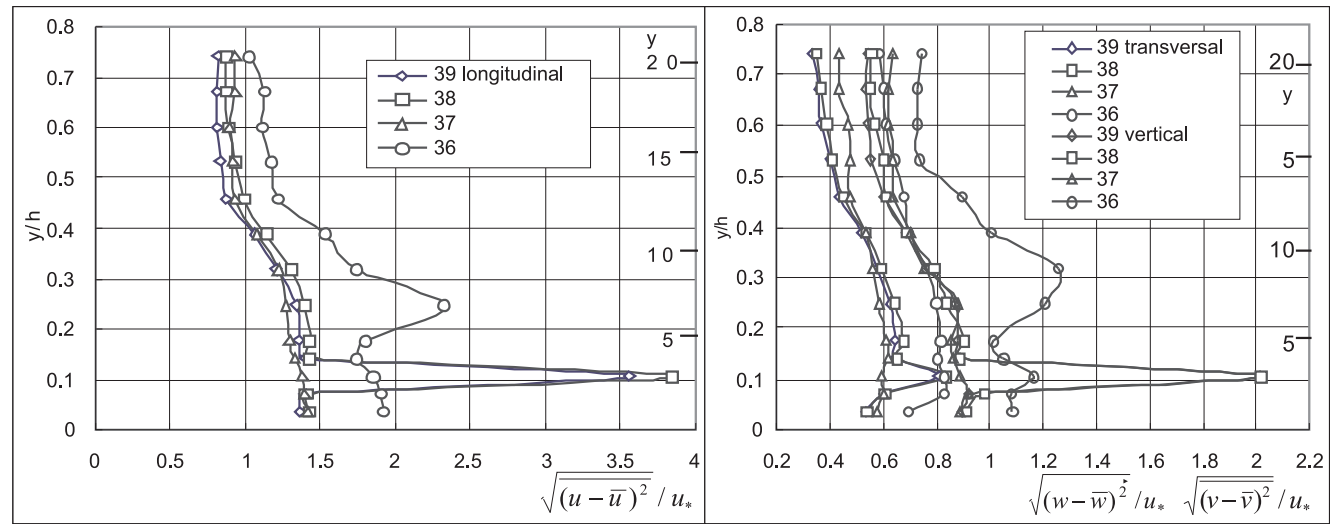

Fig. 5. Turbulence intensity profiles in measurement verticals 39, 38, 37, 36; a - of longitudinal velocities, $b$ - of transversal and vertical velocities

It is peculiar that at a height of $4 \mathrm{~cm}$ the velocity fluctuation is already much lower. Apparently, the generated eddies acquire a considerable amount of energy in a small volume, and this energy is quickly employed for turning the closest water volume. Afterwards, the eddy becomes more stable and develops slowly.

The generation of many eddies at a height of $3 \mathrm{~cm}$ ( 0.1 of water depth) takes a certain amount of energy from the flow. Therefore longitudinal and other velocity components decrease slightly at this point, which can be seen in the velocity profiles of verticals 39 and 38 (Fig. 4a, 4b). This slight decrease in velocity is not accidental, as it is obtained as an average from 9000 measurements. Consequently, all peculiarities in the velocity distribution measured in fluctuation profiles have their reasons, which can be determined.

The rapid deployment of energy received by bottom vortices as they are created means that for their further development other energy sources are necessary. This role is played by higher flow velocities and their gradients, which occur higher in the flow. The vortices are growing, as they absorb some amounts of water through fluid viscosity in their front and backsides, which lift the rotating center of the vortex. Thus absorbed water amounts with low velocities are raised to the upper part of the vortex, where they acquire energy from flow layers with a higher velocity. That is where the necessary source of energy is found. Velocity gradients are large until a height of 0.3 of water depth, where bottom vortices develop. The longitudinal velocity decreases towards the bottom because of the viscosity stress and as a result of the generation and development of initial eddies near the bottom. As a result, the profile of vertical velocity distribution and velocity gradients are formed.

Growing neighbouring vortices come into contact. Stronger ones absorb some of the volume from the weaker eddies, so that eventually these are destroyed. In the region of high velocity gradients, small eddies with a reverse rotation are generated between stronger vortices. They are visible in the PIV photos by Adrian (2000) be- 
tween the main vortices. Weaker vortices become gradually very small and are moved around the stronger ones. They are also visible in Adrian's photos between the groups of younger and older vortices.

This is how the bottom vortices are developed. It is a complex and still insufficiently investigated process. Such investigations would require a greater density of measurement points and parallel 3D measurements at neighbouring points. Then the energy balance and velocity gradients developed between vortices could be studied. For that purpose, a special device is necessary. More detailed PIV photos would be useful as well.

Adrian et al (2000) observe that the growing bottom vortices help in the generation of new ones. This probably happens when the above-mentioned central part of weaker eddies, which has not been fully destroyed, is brought by the stronger vortices down to the zone with high velocity gradients. Then they grow anew, and thus the generation process of bottom vortices continues.

The strong initial rotation of eddies may help increase their length. The ends of these growing bottom eddies turn down to the bottom and develop their rotating legs at verticals 37-36. Therefore the intensive rotation of the bottom vortices generated here is distorted and invisible (Fig. 5). This distortion decreases the intensity with which bottom vortices are generated here, so less energy is consumed for their generation and further development. Consequently, the longitudinal velocities in vertical 37 remain greater than in vertical 39 (Fig. 4a).

At a height of $3 \mathrm{~cm}$, where turbulent eddies are generated, the transversal velocity fluctuation also increases slightly. It is possible only if the axis of the generated eddy is not entirely perpendicular to the channel axis, which means that transversal flow occurs across the channel. The existence of such flow is also confirmed by the fact that the average component of transversal velocity, measured (Fig. 4b) along the whole depth of flow, is not equal to zero, At a height of $2-3 \mathrm{~cm}$ from the bottom, it is equal to $-2 \mathrm{~cm} / \mathrm{sec}$. (the minus sign shows it is directed towards verticals with higher numbers. Evidently this transversal flow is created as a result of the uneven distribution of water discharge flowing into the channel. The fluctuating transversal flow across the main channel may also be created by horizontal macro whirlpools formed throughout the width of the floodplain. These whirlpools, however, move with the flow and can raise fluctuating transversal flow only, which does not change their average intensity magnitudes. Such an insignificant asymmetry of flow is imperceptible during experiments, but can be detected by the analysis of measurement data. Nevertheless, experiments with a symmetric inflow would be necessary.

Near the sloping banks, the conditions for the generation of bottom vortices change, so that in vertical 36 all components of the velocity fluctuation are greater than in the middle flow. Apparently, because of the greater roughness of slopes, longitudinal velocities are smaller there. At a height of $7 \mathrm{~cm}(0.25 \mathrm{~h})$, longitudinal and vertical fluctuation components are also increased. This occurs at a distance of $4 \mathrm{~cm}$ from the slope, where bottom eddies are generated at the slope flow boundary. 
At a height of 9-17 $\mathrm{cm}(0.4-0.6$ of $h)$ from the bottom, time-averaged vertical and transversal velocity components change in each of verticals 36-39, and their profiles twist. This is caused by the transversal flow moving from the floodplain across the main channel. It generates longitudinal spiral vortices descending from the top of the channel slope, which increases the turbulence. The measured vertical and transversal velocities can be higher or lower, depending on the location of the measurement point in the rows of spiral vortices. The profiles of vertical and transversal velocities in vertical 36 up to a height of $10 \mathrm{~cm}(0.35 \mathrm{~h})$ twist also because of longitudinal spiral vortices created the legs of hairpin vortices, pressed to the bottom. This is why the profile in vertical 36 is very twisted.

The legs of hairpin vortices laid at the bottom create long spiral vortices, commonly called "secondary flows". (This name is not quite exact, as these long spiral vortices are only a part of the main type of bottom vortices. They are formed periodically and seem stable.)

\subsection{The Study of Macro Whirlpools}

It is characteristic of the model of a compound channel investigated here that the velocity fluctuation amplitudes of both above-mentioned vortex types are of similar size. However, the groups of bottom vortices are quite irregular, i.e. of very different length and velocity fluctuation amplitudes, which are sometimes even greater than those of macro whirlpools. Consequently, the cycles caused by macro whirlpools are well masked in many places. Therefore it was somewhat difficult to find intervals sufficiently long for an investigation with well visible cycles caused by the macro whirlpools. Fig. 6 shows the interval of measurements made in vertical 39 at a height of $15 \mathrm{~cm}$, where the cycles of macro whirlpools were less masked. The cycle length of these whirlpools is equal to about 100 measurement steps. At this height from the bottom, the interval of the whole whirlpool cycle consists of two divided parts. There are two places of minimum longitudinal velocity on the front and the backside of the whirlpool with vertical velocity components directed downwards and upwards. Two maximum velocity strips correspond to the middle of the whirlpool and to the strip between the neighbouring whirlpools (see Fig. 1).

In the interval shown in Fig. 6 the velocity fluctuations caused by groups of vortices were quite regular. At a lower height from the bottom, they were more irregular, as the bottom vortices were stronger there, so that only short intervals with 1-2-3 macro whirlpools occurred there.

Apparently because of the large irregularity of vortex groups, some contemporary mathematical models assume that the size of instantaneous velocities is accidental. Despite the above-mentioned randomness, there are some regularities which can be estimated.

As mentioned above, the fluctuation amplitudes of bottom vortices and those of macro whirlpools are of similar size in the present model. However, according to in- 


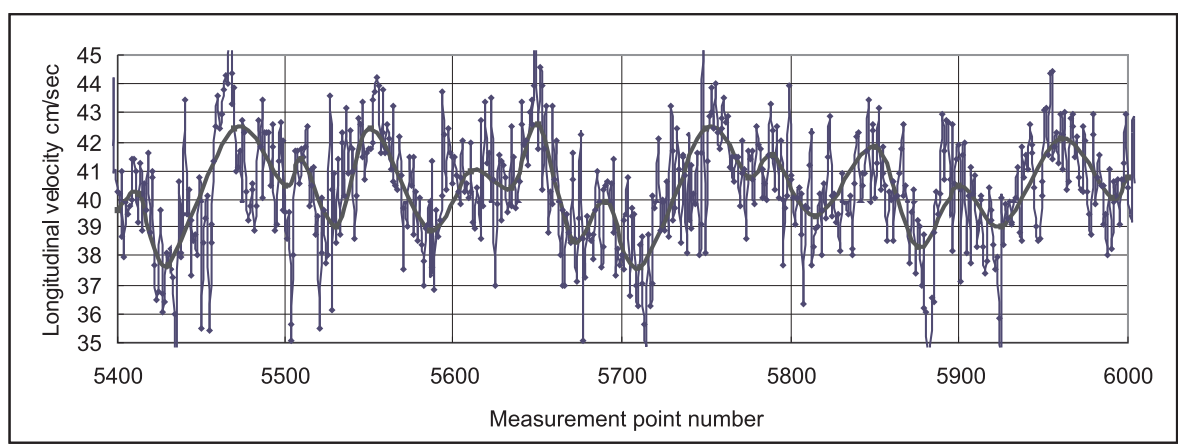

Fig. 6. Example of a longitudinal instantaneous velocity histogram with measurement points 5400-6400. The fluctuations caused by large whirlpools are illustrated with the thick line

vestigations conducted in rectangular flumes, for example by Cellino and Graf (1999), low-frequency fluctuations caused by macro whirlpools were about 4 times greater than those created by bottom vortices. This shows that the macro whirlpools are much less intensive in the compound channel investigated here. The reason is apparently the existence of the above-mentioned transversal flows (fluctuating and stable) across the main channel. They bring the growing macro whirlpools from the main channel. The length of streams flowing along the channel becomes, and therefore the macro whirlpools do not have enough time to grow fully.

In order to detect all the not entirely masked macro whirlpools by computer, the acutally existing whirlpools were compared with a standard whirlpool. For this comparison, a diagram of the standard whirlpool was moved along the velocity chronogram until the point where the standard whirlpool was similar to the one being searched for. For the latter, a correlation function (1) was calculated as:

$$
F_{j}=\frac{1}{\lambda} \sum_{i=1}^{\lambda}\left|u_{j}^{\text {stand }}-u_{j+i}\right|,
$$

where $\lambda$ - the length of the macro whirlpool in measurement time steps (for the main channel $\lambda=100), u_{j}^{\text {stand }}$ - longitudinal velocity at the current measurement point $j$ of the standard whirlpool, $u_{j+i}$ - the longitudinal velocity of the supposed whirlpool at the measurement point $j+i, i-$ the number of the chronogram point where the searched-for macro whirlpool is supposed to begin.

The sum of differences between the velocities of the standard and found whirlpools becomes minimal in the place where the standard whirlpool and the one being search for coincide. If the detected and standard whirlpools were of the same form and size, and the bottom vortices were absent, the connection function would be equal to zero.

For the purpose of constructing a standard whirlpool with a length of $\lambda$, the velocities of macro whirlpools were taken from the chronogram shown in Fig. 6.

To search for ordinary macro whirlpools, the function $F$ was calculated for each measurement point $i$, in which the beginning of the standard whirlpool was placed. 
When this point occurs at the beginning of the existing macro whirlpool, this function reaches its minimal value. Longitudinal velocity distribution in macro whirlpools is different at different heights from the bottom. Therefore standard whirlpools must be constructed for each case.

The intensity of a detected whirlpool was estimated as a relationship between the velocity amplitude of this whirlpool and the amplitude of a standard one. In order to estimate this relationship, the amplitude of the standard macro whirlpool was gradually decreased until the correlation function became minimum, i.e., until the intensities of the standard and the found whirlpools became equal.

As one can see, macro whirlpools were detected with the individual correlation function, which made it possible to detect and investigate all of them. The autocorrelation function, used in many studies, operates with the average intensity of the pairs of neighbouring vortices and is not quite suitable for this purpose.

The distribution of thus estimated intensities of macro whirlpools is shown in Fig. 7. Macro whirlpools of various intensities, mostly distorted by bottom vortices, were found almost along the whole measurement interval. They were not detected in only several places, where their calculated intensity was found very weak. Some of the detected whirlpools were unrealistically large. These whirlpools were highly distorted by bottom vortices and their groups. Along the interval shown in Fig. 6 (5500-6000 steps), where the bottom vortices are regular, the amplitudes of macro whirlpools are also regular.

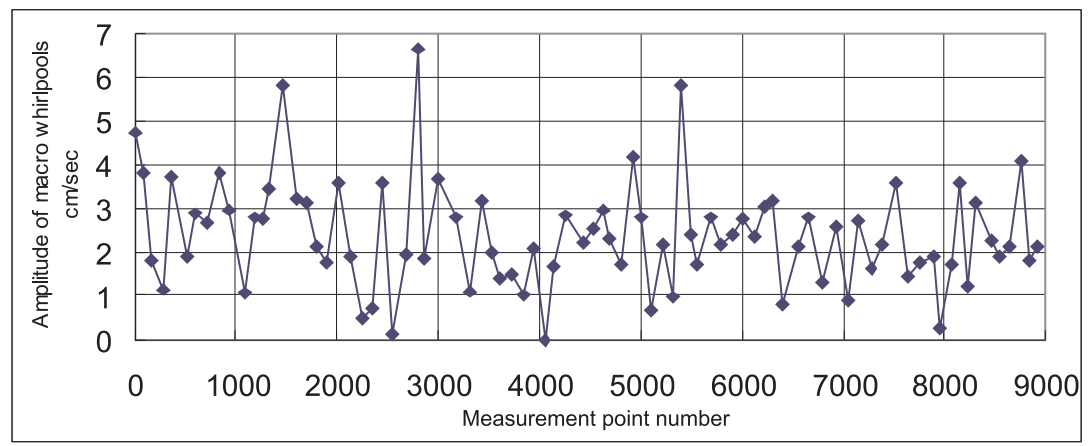

Fig. 7. Amplitudes of the longitudinal velocities of macro whirlpools in measurement vertical 39 at a height of $15 \mathrm{~cm}$

As mentioned above, under the conditions of a compound channel, macro whirlpools in the main channel are weak. As a result, the sediment transport ability of the flow is much lower than in a rectangular flume, as sediments are not lifted to the outer zone of the flow to the same extent as in a rectangular flow. This undesirable effect is increased in this model by the existence of a stable transversal flow resulting from an imperfectly symmetric inflow in the experimental channel. Transversal flows 
occur in natural flows. It is therefore useful to obtain measurements with this flow and without it.

\subsection{The Influence of Voltage Fluctuations in Electrical Systems}

The chronograms also revealed long intervals (1000-6000 measurement points) with higher or lower velocities, which were much longer than those that could be formed in the flow naturally. Such fluctuations were earlier observed in laboratories, when the water discharge produced by pumps and flowing into the channel changed because of unstable and frequency in the electrical system. It occurs in re-circulating channels. Such unnatural fluctuations distort the real velocity fluctuation amplitudes. It was necessary to eliminate them.

It was noted that these unnatural fluctuations were clearly visible only in the measurements of longitudinal velocities. Analogical changes in turbulent vortices are much smaller than those in longitudinal velocities, so they are less noticeable. This is so because some time is necessary for the development of bottom vortices until they are fully grown. Therefore, only long-lasting electrical fluctuations can have some effect, which means that the process of this influence is quite complex.

For the elimination of these distortions, the average values of instantaneous velocities in time intervals of estimated length were calculated. These values were changing about the average value over the whole measurement time. To obtain the exact average meanings of flow velocities under such conditions, a relatively long measurement time (6.0 $\mathrm{min}$ ) was necessary. The corrections were calculated for each measurement point with this point in the middle of the time interval used for averaging.

The length of the averaging intervals must not be shorter than the really existing cycle in natural flow velocity fluctuations. The longest fluctuation cycle in the main channel is caused by the macro whirlpools. Their cycle in the main channel is 100 measurement steps long. Consequently, the time interval for averaging here must not be shorter than 100 steps.

However, horizontal macro whirlpools are also formed throughout the entire width of the floodplain. They can create transversal velocity fluctuations in the main channel, as well, which also move along the flow. The cycle of horizontal whirlpools in the floodplain is longer. The width of the floodplain is $60 \mathrm{~cm}$, so the distance between vortices of this type is $60 \times 6=360 \mathrm{~cm}$. The average flow velocity in the right floodplain is $31 \mathrm{~cm} / \mathrm{sec}$, so the length of cycle is $360 / 31=11.6 \mathrm{sec}$, or $11.6 \times 25=290$ measurement steps. It is the longest cycle that can be formed in this model, as larger flow elements containing macro whirlpools are absent. To avoid the elimination of these fluctuations, the above-mentioned averaging interval for the valley flow must be equal to 300 measurement steps.

On the left side of the floodplain, the water discharge and flow velocities are slightly lower than on the right side. Therefore, the horizontal whirlpools move with slightly different velocities, and their position along the flow is somewhat different. 
Consequently, the fluctuating flow velocities are formed across the main channel. In the case of symmetrical floodplain flows, the flow across the main channel would be impossible. Thus, the fluctuations of transversal velocities of about 300 measurement steps in length are natural and visible in chronograms. This fluctuation of transversal velocity changed the conditions for the development of macro whirlpools. That is why their amplitudes and the size of longitudinal velocities fluctuated at the same frequency. Consequently, for the calculation of corrections the averaging length in the valley and the main channel was set at 300 steps. The calculated corrections are plotted in Fig. 8. Measurement data were corrected in this way.

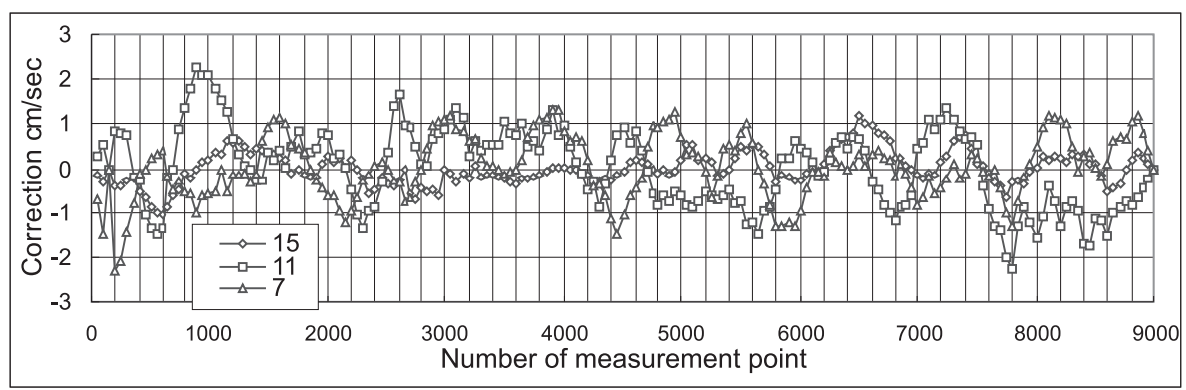

Fig. 8. Longitudinal velocity corrections eliminating the influence of electrical voltage fluctuations in vertical 39 at heights of $7-15 \mathrm{~cm}$ from the bottom

The largest longitudinal velocity correction was over $2 \mathrm{~cm} / \mathrm{sec}$. In these intervals the amplitudes of the measured velocity fluctuations were mostly $4-6 \mathrm{~cm} / \mathrm{sec}$. Thus the errors in these intervals were as large $50 \%$. Differences in the average fluctuations of longitudinal velocities calculated with corrected and uncorrected measurement data reached $5 \%$. These errors are fairly significant, so they need to be eliminated by this procedure. Such distortions could be avoided by means of an apparatus for electrical voltage stabilization or by means of upper leveling reservoirs. When they are absent, a parallel measurement of flow velocity at the beginning of the channel would be helpful. It could be directly used for a more exact calculation of corrections. It is possible that the electrical voltage twisting cycles have a similar length as the ones occurring in the water flow. Such measured velocities could be used for calculations to eliminate these unnatural fluctuations.

\subsection{Development of Bottom Vortices}

As mentioned above, the high gradients of longitudinal velocities in the main channel lead to the formation of hairpin-like bottom vortices. They occur in groups.

When the first vortex in the group is formed, it decreases the longitudinal velocity near the bottom in that place, which is favourable for vortex generation. When the first vortex is moved by the flow, another vortex can be formed in its place. As a result, vortex groups are formed. This process continues until it is stopped by some reason, 
for instance, because of: (1) the older group of vortices going on higher, (2) the passing rising part of a macro whirlpool, which lifts the vortex group into the outer zone of the flow, (3) any accidental event changing the velocity.

Below the already growing group of bottom vortices, a zone of lower longitudinal velocities is formed. It decreases velocity gradients near the bottom. Owing to the decrease in longitudinal velocity below the row of vortices, a zone of almost constant longitudinal velocities is formed between the neighbouring groups. This phenomenon has been observed by many researchers.

In order to observe vortices passing the measurement points in the central verticals of the main channel, it is most convenient to analyze vertical velocity chronograms. When the front of a vortex approaches, velocities are directed downwards. Here their minimum (negative maximum) is found. When the end of the vortex passes by, the velocities are directed upwards. Thus the chronogram becomes twisted. The number of twists shows how many vortices have been recorded by the measurement. In the interval of 100 points, 28-32 vortices passed by. An arriving vortex group also has the minimum values of the longitudinal velocity. When a vortex group situated above passes by, it decreases the longitudinal velocity. The maximum is formed when a neighbouring vortex passes below. After this, the velocity graph is slightly twisted, as the vortices further from the measurement point pass by. The longitudinal velocity decreases gradually until the next vortex group approaches. When a vortex of any group is passing by the measurement point with its inner part, its longitudinal velocity changes slowly.

Most of the vortices flowing not too far from the measurement point only cause twists in chronograms. However, hardly any of them pass this point with their central part. These vortices can serve for the study of their characteristics. When the central part of a vortex passes near the measurement point, vertical velocities change significantly, but there is much less change in longitudinal pulsations. The observation of these velocities made it possible to recognize the searched-for vortices. For this search, it is necessary to estimate changes in vertical the longitudinal velocities at two neighbouring measurement points. After some trials, it was assumed that a vortex whose central part passes the measurement point is detected when the vertical velocity grows by not less than $0.5 \mathrm{~cm} / \mathrm{sec}$ and the change in the longitudinal velocity is less than $0.5 \mathrm{~cm} / \mathrm{sec}$. When the velocities selected for this purpose were, for example, 0.6 and $0.4 \mathrm{~cm} / \mathrm{sec}$, stronger vortices were detected. When the center of a vortex passes the measurement point at a greater distance, the change in the longitudinal velocity is greater. In the lower or the upper part of a vortex, longitudinal velocities change the most while vertical velocities change slowly. To catch a vortex in both these cases, the control increment in vertical velocity was set at $0.2 \mathrm{~cm} / \mathrm{sec}$, and of longitudinal velocity at less than $0.7 \mathrm{~cm} / \mathrm{sec}$.

It is a well known fact that the central part of a vortex rotates statically, and the velocities are proportional to the radius. From these conditions it was possible to calculate the distances of the detected vortex center from these two points, and the 
rotational velocity of the vortex. These two calculated rotational velocity values were somewhat different because the trajectories of the vortex points were not quit circular, or the rotation of the central part was not quite static, or the two measurement points could not be placed in one vortex.

From the measurements at various points along the height of verticals, the relationship between the rotational velocity and the distance from the bottom was estimated.

Naturally, the method described above made it possible to detect only sufficiently large vortices, in which two neighbouring measurement points could be placed. The distance between measurement points was $1.2-1.5 \mathrm{~cm}$. It sometimes happened that rotation measured at these two points was very different or even. If the difference was greater than $50 \%$, the points were rejected. This usually happened when the vortex was too small for such an investigation. During measurements with all 9000 steps, 300-400 sufficiently large vortices were found. These were mostly vortices of the groups flowing through the measurement point. Having emerged and developed under very different and random conditions, these vortices had significantly different intensities of rotational velocities. The calculated rotational velocities were disposed according to the turning intensity, and so the probability graphs of this characteristic were obtained. They are plotted in Fig. 9. As can be seen, the change in rotational velocities (30-70\% probability) at different heights from the bottom is similar to the change in the fluctuations of vertical and longitudinal velocities (Fig. 5). It is quite natural, as the rotational velocities of vortices are proportional to vertical and longitudinal velocities.

The change in the rotational velocity of a vortex at heights of 5,7 , and $9 \mathrm{~cm}$ is larger than at greater heights, as velocity gradients are large there. At heights of 11, 13 , and $15 \mathrm{~cm}$ vortices are not developed further. They are lifted by macro whirlpools, so that their rotational velocity differs less.

The distances calculated from the measurement point to the centers of the vortices being searched for are in many cases quite large. They show the possible sphere of influence of these vortices. It was interesting to estimate how far this influence reached in various directions. In the direction of the flow, their influence is naturally limited by the distance between them, Downwards and upwards, however, this influence is limited only by their intensity. The sphere around the measurement point was divided into 12 sections, each of 300 . The sections 1 and 12 were directed downwards. Sections 1-6 were at the left side of vortices. The calculation results are plotted in Fig. $10 \mathrm{a}$ and $10 \mathrm{~b}$.

Fig. 10a shows the number of vortices that occurred in the sections. The measurement results at heights of 5, 9 and $15 \mathrm{~cm}$ from the bottom are presented. Mostly the vortices are directed downwards (sections 1 and 12) and upwards (sections 6 and 7). The cases of horizontal directions occur seldom, as these are few in these directions, and the distances between them are relatively short. In Fig. 10b the distances from the measurement point to the center of the vortex are shown. 


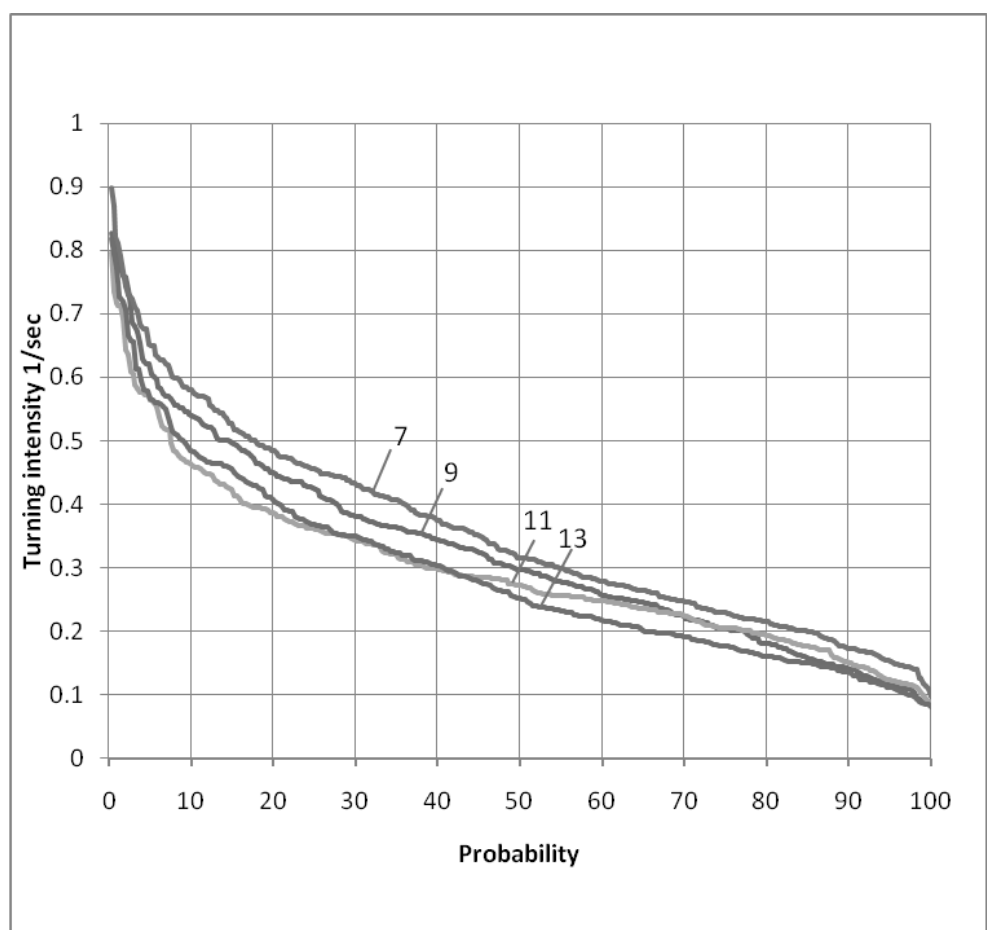

Fig. 9. Probability of the turning intensity of bottom vortices in measurement vertical 39 at heights of 7 to $13 \mathrm{~cm}$ from the bottom
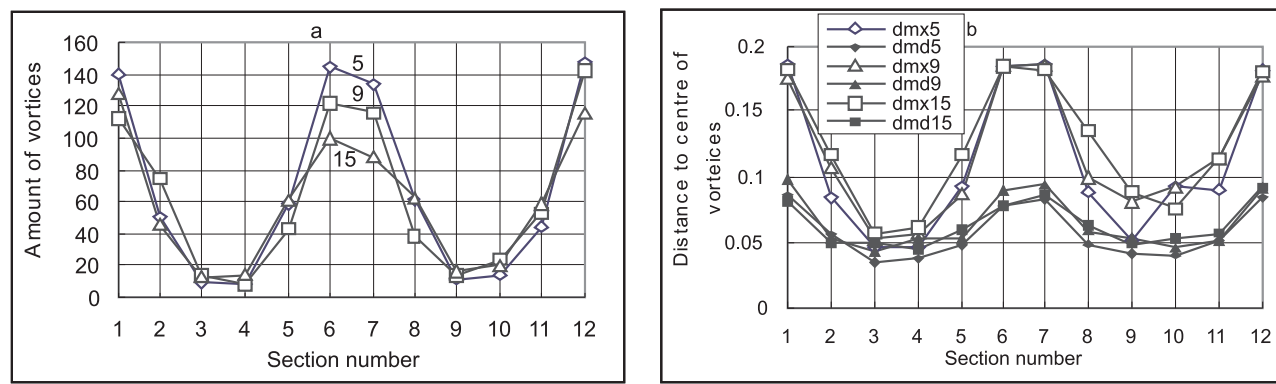

Fig. 10. The number of vortices which occurred in particular sections (Fig. a) at heights of 5, 9 , and $15 \mathrm{~cm}$ from the bottom. The distances of the vortices from the measurement point (Fig. b): dmx5, dmx 9 and dmx15 - the maximum distances of the vortices from the measurement point at heights of 5, 9, $15 \mathrm{~cm}$; dmd5, dmd, dmd 15 - the middle distances of the vortices from the measurement point

The distances between the vortices in developed groups are not large. Therefore only the oldest vortex can freely absorb water volumes by fluid viscosity. These volumes are brought down to the region of a neighbouring vortex, which sends this water volume to the next, younger vortex. Brought down, these volumes lift the vortex group higher. The energy of vortices is expended on this process, but this energy is 
restored by an upper flow layer with higher velocities. Here, the rotation of the vortex is increased by fluid viscosity. By influence of vortices, the flow in this upper layer becomes somewhat twisted. Therefore our method would make it possible to register the influence of vortices in this layer, as well. The extent of this influence upwards and downwards appeared quite similar.

As can be seen, groups of bottom vortices operate in unison, which is why they are called "coherent structures". According to PIV photographs (Adrian et al 2000), these structures are usually not quite regular. In some places, the distances between vortices are larger. Apparently this occurs because the weaker ones have been destroyed. Through these larger intervals, water volumes are brought downwards, and these new vortices are lifted higher than the older ones. As a result these structures become irregular.

As already mentioned, the rotation of the vortex and velocity fluctuations would be damped out if they were not supported by velocity gradients along the flow depth. The energy of flow layers with higher velocity is employed for that purpose. In this way the stability of macro whirlpools is supported, as well. The analysis of the distribution of instantaneous velocities showed the process of the formation and further development of bottom vortices, Macro whirlpools, however, were already formed in the measurement section, and the process of their generation could not be observed. It is safe to assume that at the beginning of the experimental channel, where the turbulence is still developing, the largest bottom vortices continue to grow and thus assist the formation of macro whirlpools. However, for the full development of macro whirlpools, the profile of longitudinal velocities must be formed.

\section{The Analysis of Turbulent Vortices in the Valley}

At the flow boundaries of the valley, where high velocity gradients are formed, turbulent vortices are generated as well, similar to those in the main channel. At the banks of the valley horizontal vortices are generated, too. These can also be divided into two kinds of structures: bank vortices (and their groups) and horizontal macro whirlpools, which take up the whole width of the valley side. The influence of all these vortices intertwines, which makes it quite difficult to estimate properly their parameters.

\subsection{Vertical Vortices in the Valley}

For the study of vertical vortices in the valley, the chronograms of vertical velocity components are most convenient. Horizontal vortices do not influence these components, as they contain only longitudinal and transversal velocity components. Vertical velocities in the valley are formed only by vertical vortices, so they can be investigated in these chronograms much easier than in longitudinal and transversal ones. In the valley, as in the main channel, the influence of bottom vortices and their groups and of macro whirlpools is reflected in chronograms. The influence of macro whirlpools 
is therefore somewhat masked. However, there are many intervals, where bottom vortices and their groups are quite regular and macro whirlpools are well visible. Such an interval, between measurement points 2900 and 3450, is illustrated in Fig. 11, which shows measurements in vertical 19 at a height of $4 \mathrm{~cm}$.

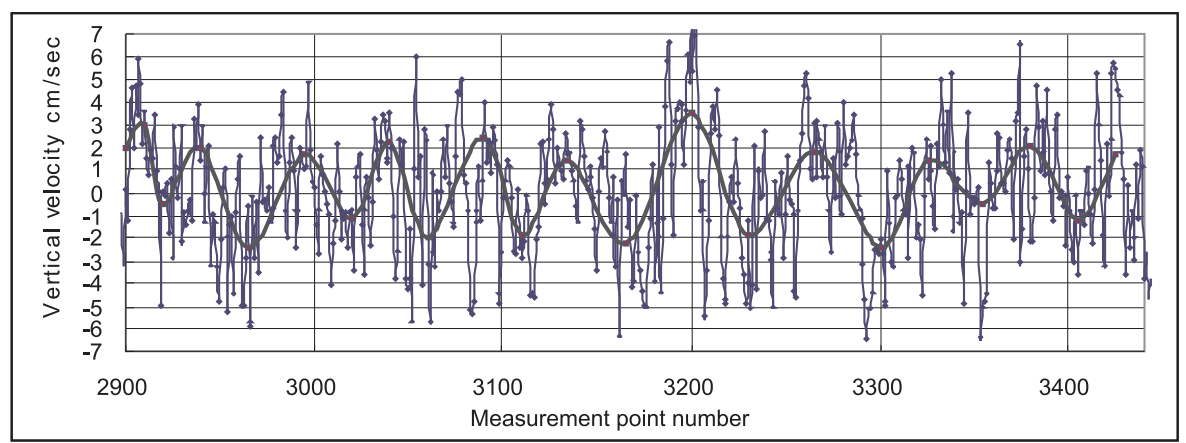

Fig. 11. Vertical bottom vortices (thin line) and macro whirlpools (thick line) in the valley (vertical 19)

Since water depth in the valley was $h=12.3 \mathrm{~cm}$, the length of macro whirlpools amounted to $L=6 \times h=73.8 \mathrm{~cm}$. The average velocity in the valley was $31 \mathrm{~cm} / \mathrm{sec}$. Then the time in which macro whirlpools flowed by the measurement point was $t=$ $L / v=73.8 / 31=2.4 \mathrm{sec}$, or $2.4 \times 25=60 \mathrm{steps}$, in measurement points. In Fig. 11, the average length of macro whirlpools is also about 60 steps. This length changes slightly along the flow because the longitudinal velocity components of vertical macro whirlpools are changed by horizontal macro whirlpools, which make the flow twist. Then the direction and flow velocity of vertical macros changes, as well. Consequently, the length pulsation of vertical macro whirlpools is of the same frequency as that of horizontal macro whirlpools.

The bottom vortices in the valley are also hairpin like. As a result, their legs, laid at the bottom, cause longitudinal spirals (secondary flows) with increased fluctuations of transversal velocities.

It should be noted, that macro whirlpools in the main channel are much less visible in the chronograms of vertical velocities than in those of longitudinal velocities because they are masked by bottom vortices. In the valley, as shown in Fig. 11, the fluctuation of bottom vortices and their groups are also large, even somewhat larger than the fluctuation of macro whirlpools. However, the bottom vortices in the valley are much more regular than in the main channel, where their regularity is distorted by longitudinal spiral vortices, caused by transversal flow across the channel.

The intensity of vertical macro whirlpools in the valley was also studied with the use of correlations calculated according to formula (1). The calculated amplitudes of macro whirlpools in the valley are plotted in Fig. 12. The same figure also shows the amplitudes of macro whirlpools calculated for vertical 39 in the main channel. 
They are even somewhat smaller than those in the valley, as their growth is limited by transversal flow across the channel.

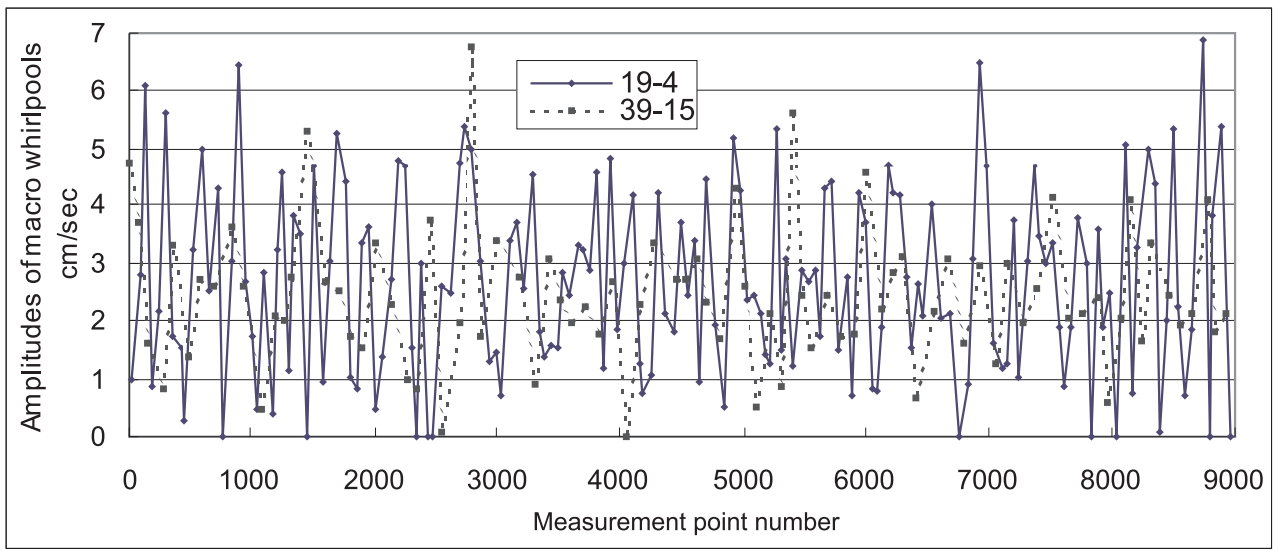

Fig. 12. Amplitudes of the vertical velocities of macro whirlpools in vertical 19 at a height of $4 \mathrm{~cm}$ and in vertical 39 at a height of $15 \mathrm{~cm}$

It is easy to see that the influence of bottom vortices in the valley also distorts the intensity of macro whirlpools, and as a result they get dispersed. However in many intervals, fluctuation cycles of about 150 measurement steps are clearly visible. Such fluctuation is caused by horizontal macro whirlpools is valley. Their cycle is 290 steps. Flowing once through the measurement point, they make the direction of flow parallel to the channel axis twice (each 150 steps). Then the conditions for the development of vertical macro whirlpools apparently improve. When the flow is diagonal, these macro whirlpools become weaker. Consequently, this fluctuation cycle of 150 steps is formed.

\subsection{Horizontal Vortices in the Valley}

At the bank boundary of the flow, where large longitudinal velocity gradients are formed, turbulent vortices are generated in a similar way as those at the bottom. Because of the large width of the valley, they are accordingly larger. The largest bottom vortices reach a height of 0.3 of water depth. Analogical valley vortices could be as large as 0.3 of the valley width, i.e. $0.3 \times 60=18 \mathrm{~cm}$. The average valley flow velocity is $0.3 \mathrm{~cm} / \mathrm{sec}$. Such a vortex flows by the measurement point in $18 / 30=0.6 \mathrm{sec}$, i.e. $0.5 \times 25=15$ steps. These vortices also form groups of about 100 steps in length. The distance between horizontal macro whirlpools, as already mentioned, is equal to 290 measurement steps. All these vortices are represented in the chronograms of measured velocities. A characteristic interval of a longitudinal velocity chronogram, containing fluctuations caused by vertical and horizontal vortices, is plotted in Fig. 13. 


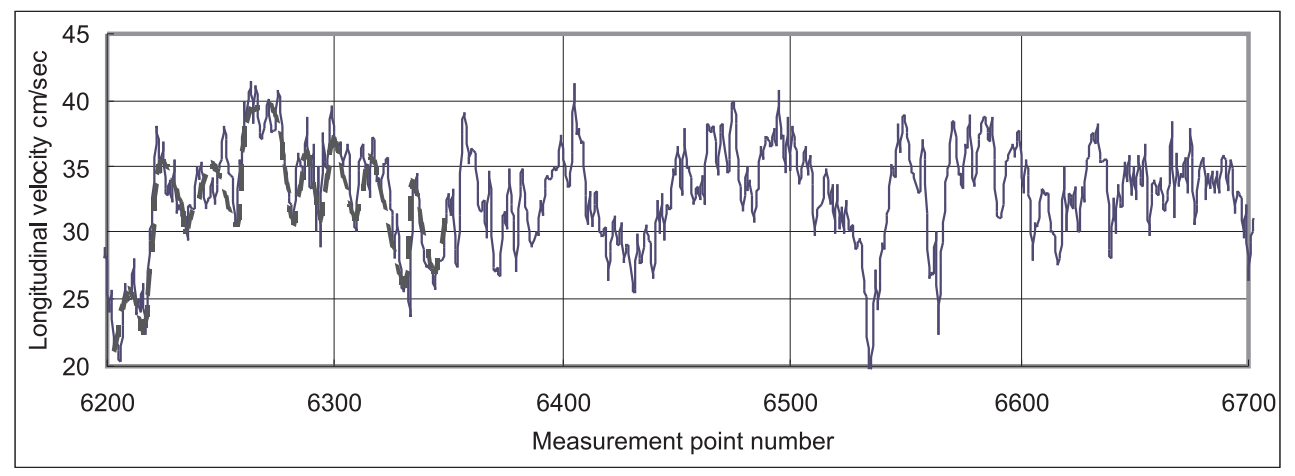

Fig. 13. Longitudinal velocities in vertical 19 at a height of $4 \mathrm{~cm}$ caused by horizontal and vertical vortices in the valley. The dotted line illustrates single horizontal vortices

In this figure it is possible to observe one long twist caused by a horizontal macro whirlpool in the interval 6220-6540 of the chronogram. The finest fluctuations are caused by bottom vortices and their groups. Single horizontal vortices (dotted line) cause fluctuations of 10-30 steps; fluctuations caused by groups of vortices are up to 60-120 steps long. There are some intervals in which single horizontal vortices are not observed. In these places, they are either weak or masked by groups of bottom vortices.

The fluctuation amplitude of longitudinal velocities observed in the chronogram, caused by single horizontal vortices, reaches $5-10 \mathrm{~cm} / \mathrm{sec}$. Amplitudes of horizontal macro whirlpools amount to $15-20 \mathrm{~cm} / \mathrm{sec}$.

\section{Vortices at the Contact of Main Channel and Valley Flows}

At the contact of the main channel flows and valley flows, the gradients of longitudinal velocity, critical for the generation of vortices with vertical axes, are also formed. The vortices formed here are also of two main types. They are either small contact vortices, generated at points of critical velocity gradients, or large macro whirlpools, supported by longitudinal velocity gradients across the channel width. Horizontal vortices, generated in this manner, cause longitudinal and transversal velocity fluctuations. The insignificant components of vertical velocity exist only because the vertical axis of macro whirlpools is slightly bent by longitudinal velocities growing along the water depth. Vertical velocity components here are mainly due to bottom vortices. Here they are also hairpin like. Therefore they have all three velocity components. They are quite strong and can also mask the above-mentioned horizontal macro whirlpools. A characteristic interval of measurements in vertical 30 at a height of $4 \mathrm{~cm}$, where the vortices are clearly visible, is illustrated in Fig. 14. It shows longitudinal and transversal velocity fluctuations.

The thin lines in both chronograms show high frequency fluctuations caused by bottom vortices. In Fig. 14a a macro whirlpool is visible at an interval of 10-160 mea- 

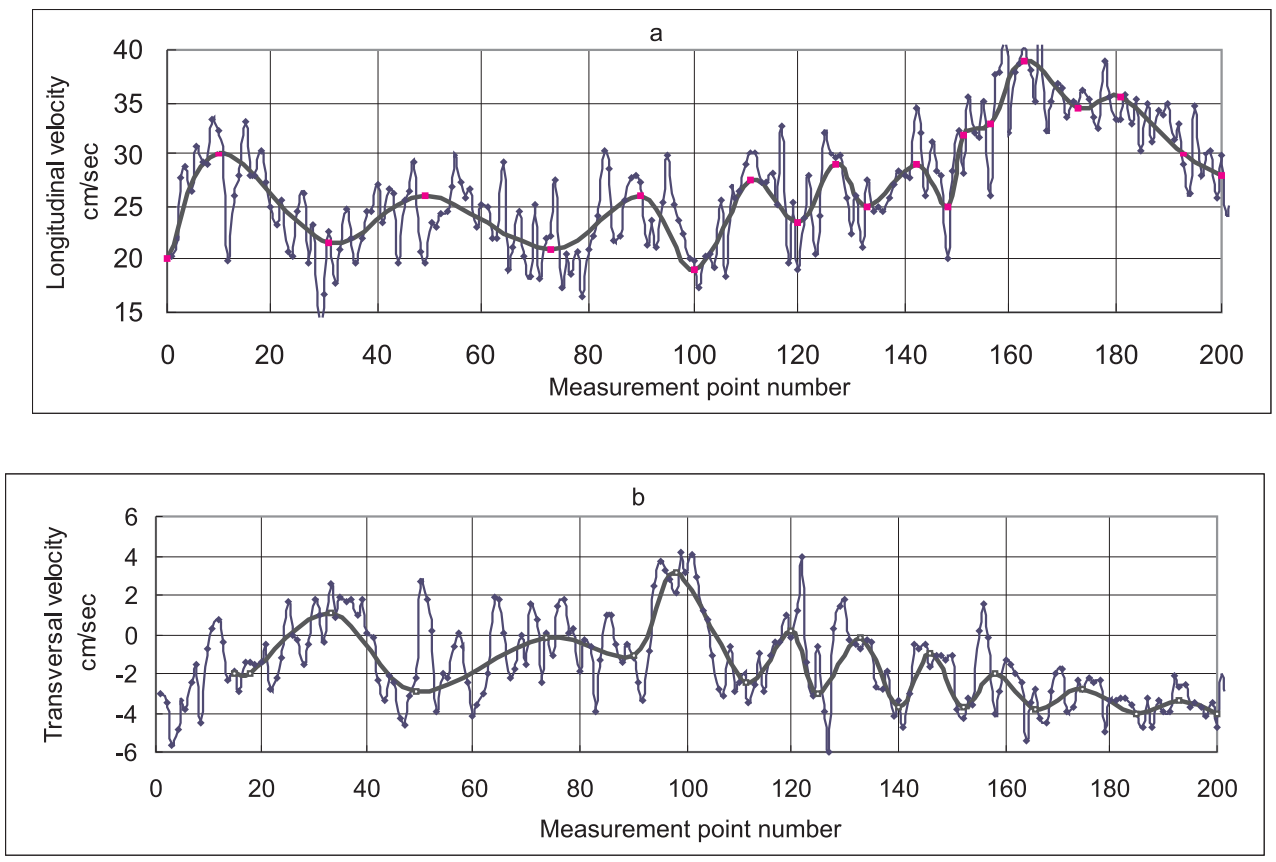

Fig. 14. Longitudinal velocities (a) and transversal velocities (b) in vertical 30 at a height of $4 \mathrm{~cm}$. The thin lines depict velocities caused by bottom vortices, and the thick lines represent velocities caused by horizontal contact vortices in the main channel

surement points. Its length is equal to 6 distances to the middle of the channel. The cycle length of horizontal vortices in a row at the contact of flows changes between steps 15 and 50 (thick line). These lengths of longitudinal and transversal velocities are the same; only the place with maximum longitudinal velocities corresponds to the minimum of transversal velocities. High velocities in channel flow increase longitudinal velocities in the valley at the contact with the main channel. This increases the turning intensity of horizontal whirlpools in the valley.

The fluctuation amplitude of longitudinal velocities in macro whirlpools is equal to about $10 \mathrm{~cm} / \mathrm{sec}$, and that of vertical velocities to about $3 \mathrm{~cm} / \mathrm{sec}$. Amplitudes of fluctuations in the vortices in a row at the contact of the two flows are about $6 \mathrm{~cm} / \mathrm{sec}$ for longitudinal velocities and $4 \mathrm{~cm} / \mathrm{sec}$ for transversal velocities. These amplitudes are much larger than the amplitudes of macro whirlpools generated at the bottom, which are low in this compound channel.

\section{Possibilities of Mathematical Modelling}

Flow structures are also investigated by mathematical modeling. Mathematical modeling of actually existing flow structures and the development of hairpin vortex groups was performed by Adrian et al (2000). The 3D Direct Numerical Simulation (DNS) was employed for this purpose. For the calculation of these hairpin-like vortices, their 
initial generation was included in the model. Further development of hairpin vortices was calculated according to Navier-Stokes equations. To estimate, for example, the existing macro whirlpools, it would also be necessary to include their corresponding initial conditions in the model. As a result, the development and the influence of these whirlpools on the flow structure would be estimated as well. In addition, such models could also include the calculation of sediment motion and deposition. Thus they would provide more exact results than contemporary models. Instead of building physical models, one could employ such mathematical models to investigate, for example, several model variants with various relative measurements.

The difficulty of employing DNS for such investigations lies in a proper selection of initial conditions and in the long time of computer work. That is why simpler models have been created, which calculate only time-averaged velocity values. They are less precise. Their exactness depends on the methods employed to estimate the existence of turbulence. Various methods are created for this purpose, including easily defined algebraic equations. The exactness of these methods may be increased through research on the development of actually existing turbulent vortices.

\section{Conclusions}

In the compound channel investigated here, two kinds of turbulent vortices were detected: hairpin-like bottom vortices and macro whirlpools. Vertical and horizontal macro whirlpools occurred both in the main channel and in the valley. The chronograms of measured velocities were used to calculate the characteristics of both these kinds of turbulent vortices.

The analysis of instantaneous velocity measurements revealed that bottom vortices did not receive the amount of energy sufficient for their growth as they were generated. They owed their further development to longitudinal velocity gradients. They obtained the necessary energy from higher flow layers of greater velocities. Macro whirlpools were also supported by these velocity gradients.

Macro whirlpools in the main flow of the compound channel were much weaker than those in rectangular flumes because of a transversal flow running through the main flow. This reduced the sediment transport ability of the channel.

The velocity values measured in re-circulating channels can be distorted by the fluctuations of voltage in the electrical system. In such cases the pumps produce a fluctuating water discharge. These distortions can be eliminated by a special calculation procedure.

A detailed analysis of turbulent vortices can help to better understand the processes taking place in the flow and to improve the methods of studying them. The analysis of these measurement data is continued with the aim of finding better ways to estimate the characteristics of detected vortices. 


\section{Acknowledgments}

I am particularly indebted to Prof. W. Czernuszenko for granting access to his most recent data on the measurement of turbulence in a water laboratory in Warsaw, as well as for useful discussions.

\section{References}

Adrian R. J., Meinhart C. D., Tomkins C. D. (2000) Vortex organization in the outer region of turbulent boundary layer, J. Fluid Mech., 422, 1-54.

Albayrak I., Lemmin U. (2007) The effect of water depth on the dynamics of secondary currents in turbulent open-channel flow over rough bed. River Flow 2008, Proceedings of the international conference on fluvial hydraulics, Turkey, September 3-5, 2008, 271-279.

Albayrak I., Lemmin U. (2008) A study of open-channel surface flow dynamics using Large Scale Particle Image Velocimetry (LSPIV). River Flow 2008, Proceedings of the international conference on fluvial hydraulics, Turkey, September 3-5, 2008, 195-202.

Bravo H. R., Meinecke J. W. (1997) Entrance flow and the achievement of uniform fully developed open channel flow, Proceedings 27th Congress IAHR, Theme A, San Francisco, CA, 726-728.

Breuer M., Haenel D. (1989) Solution of the 3-D incompressible Navier-Stoces equations for the simulation of vortex breakdown, Proc. of 8th GAMM conf., 29, 42-51.

Cellino M., Graf W. H. (1999) Sediment-laden flow in open-channels under non-capacity and capacity conditions, Am. Soc. Civ. Eng., J. Hydr. Engr., 125 (5), 455-462.

Cuthbertson A. J. S., Ervin D. A. (1999) The interaction between turbulent vortices and fine sediment particles - a possible reason for enhanced settling characteristics? Proc. 28th IAHR Congr., Graz, Austria, CD-ROM.

Czernuszenko W. (2002) Turbulent shear stresses and prime velocity distribution in compound channels, Archives of Hydro-Engineering and Environmental Mechanics, 49 (3), 3-17.

Czernuszenko W., Kozioł A., Rowiński P. (2007) Measurements of 3D turbulent structure in a compound channel, Archives of Hydro-Engineering and Environmental Mechanics, 54 (1), 3-21.

Czernuszenko W., Rowiński P. (2008) Reynolds stresses in compound open channel flow-flume experiments. River flow 2008, Proceedings of the international conference on fluvial hydraulics, Turkey, September 3-5, 2008, 289-297.

Grishanin K. V. (1969) Dynamics of River Flow, Gidrometeorologicheskoe Izdatelstvo, Leningrad, 428 (in Russian).

Klaven A. B. (1966) Investigation on the Structure of Turbulent Flow, Trudy Gosudarstvennovo Gidrologicheskovo Instituta, Vypusk 136, 65-76 (in Russian).

Nezu I., Azuma R. (2004) Turbulence characteristics and interaction between particles and fluid in particle-laden open channel flows, Journal of Hydraulic Engineering, 130 (10), 988-1001.

Nezu I., Nakahawa H. (1993) Turbulence in open channels, IAHR monograph series, A. A. Balkema. Rotterdam, The Netherlands.

Rimkus A., Vaikasas S. (2001) The length of laboratory channels necessary for the stabilization of suspension flow, Water Management Engineering Transactions, 18 (40), Lithuanian Institute of Water Management. 59-64.

Robinson K. (1991) Coherent motions in the turbulent boundary layer, Fluid Mech., 23, 601-639.

Rodriguez J. F., Garcia M. H. (2008) Laboratory measurements of 3-D flow patterns and turbulence in straight open channel with rough bed, Journal of Hydraulic Research, 46 (4), 454-465.

Vanoni V. A. A., Nomicos G. N. (1959) Resistant properties of sediment-laden streams, J. Hydr. Div. Am. Soc. Civ. Engrs., 85 (HY5). 
Widera P., Ghorbaniasl G., Lacor Ch. (2009) Study of the sediment transport over flat and wavy bottom using large-eddy simulation, Journal of Turbulence, 10 (33), 1-20.

Yalin M. S. (1992) River Mechanics, Pergamon Press, Oxford, England. 